\title{
EFFECT OF ENVIRONMENT ON PHAGOCYTOSIS OF RABBIT SPERMATOZOA*
}

\author{
J. M. BEDFORD \\ Department of Physiology, Royal Veterinary College, London, $\mathcal{N} . W .1$
}

(Received 15th October 1964)

\begin{abstract}
Summary. The phagocytosis of rabbit spermatozoa has been studied with the electron microscope, and the process of ingestion of both intact and damaged spermatozoa by leucocytes in the oestrous uterus is described. The cell membrane and acrosome cap remained intact in many sperm heads wholly ingested by uterine leucocytes up to $12 \mathrm{hr}$ after mating. However, only spermatozoa with damaged acrosomes and/ or damaged head membranes were ingested to any extent in the pseudopregnant uterus, in the pleural cavity, or following in-vitro incubation with leucocytes obtained from the oestrous uterus, intact sperm heads merely adhering to the leucocyte surface. It is suggested that some change occurs in the surface membrane of spermatozoa in the oestrous uterus which renders the intact sperm head acceptable to uterine leucocytes. Such surface changes may be associated with the process of sperm capacitation.
\end{abstract}

\section{INTRODUCTION}

It has been known for many years that spermatozoa may be ingested by uterine leucocytes in the period following mating (Austin, 1957). Not until 1951, however, was it shown that an important functional change, termed capacitation, occurs in spermatozoa during passage through the female tract (Austin, 1951; Chang, 1951). Fertilization cannot occur before the process of capacitation is completed, since non-capacitated spermatozoa are apparently not competent to penetrate the zona pellucida. The nature of the change which occurs in the spermatozoon during capacitation remains uncertain.

Evidence has been presented which suggests that the metabolic pattern of rabbit spermatozoa changes during uterine incubation (Hamner \& Williams, 1963; Mounib \& Chang, 1964); however the association of such changes with the capacitation process remains yet to be established. Light microscope studies on rabbit spermatozoa recovered from the uterus failed to reveal structural changes in the sperm head which might be interpreted as being a concomitant of the capacitation process (Adams \& Chang, 1962; Bedford, 1963; Austin, 1963). Electron microscope studies likewise failed to reveal any consistent change in the fine structure of the head in uterine spermatozoa (Bedford, 1964). In the latter study it was evident nevertheless that although the plasma

\footnotetext{
* Presented at the Annual Meeting of the Society for the Study of Fertility, Oxford, July 1964.
} 
membrane of ejaculate spermatozoa, typically swollen over the flat surface, usually remained adherent to the periphery of the acrosome, this membrane had become completely divorced from the underlying acrosome and remained adherent only to the post-nuclear cap in many uterine spermatozoa. Bearing in mind methods of preparation of spermatozoa for electron microscopy, it seemed difficult to accept with confidence the possibility that such a difference in the disposition of the head plasma membrane of free spermatozoa might have some physiological significance, except that complete separation of the plasma membrane away from the acrosome surface was commonly seen also in those intact sperm heads which had been partially or completely engulfed by uterine leucocytes (Pl. 1, Fig. 4 and Pl. 2, Fig. 5). If in intact ingested spermatozoa the cell membrane were present in close apposition to the underlying acrosome in vivo, one would expect that, during fixation, the mass of the encompassing leucocytes would act to prevent the distortion of the membrane seen so typically in free spermatozoa. However, as the membrane was usually completely separate from the underlying acrosome in the intact ingested sperm heads, it seemed reasonable to regard the loose arrangement of the plasma membrane in these ingested spermatozoa as representing the actual disposition of the membrane in vivo. At this point it was clearly of interest to examine also the disposition of the surface membrane of non-capacitated sperm heads, after phagocytosis.

This report describes firstly the normal mode of phagocytic ingestion of intact and damaged spermatozoa in the oestrous uterus during the $12 \mathrm{hr}$ after mating. Secondly, in partial contrast to the process occurring in the oestrous uterus, it is shown that only damaged sperm heads are ingested in the pseudopregnant uterus, in the pleural cavity, or during in-vitro incubation with uterine leucocytes. In these cases, the intact sperm head is never ingested to any extent and remains free or merely adherent to the surface of the leucocyte.

\section{MATERIALS AND METHODS}

Adult domestic rabbits of mixed breed were used. Uterine contents were obtained from a total of sixteen oestrous females 10 to $12 \mathrm{hr}$ after natural mating with fertile males, by flushing the uterine horns with $2.0 \mathrm{ml}$ Ringer solution containing $25 \%$ heated fresh serum.

To obtain uterine leucocytes for in-vitro incubation experiments, a suspension of sterile animal charcoal in $0.2 \mathrm{ml}$ Ringer solution was first instilled via the cervical canals into each uterine horn of six oestrous females. Between 6 and $7 \mathrm{hr}$ later the uterine horns were flushed with $2.0 \mathrm{ml}$ serum-Ringer solution. The numbers of leucocytes harvested by this method varied considerably between animals. In three experiments ejaculate spermatozoa washed free of seminal plasma were used for incubation with the leucocyte suspensions at a concentration of between two and four spermatozoa per leucocyte. In three experiments, spermatozoa obtained directly from the vas deferens were added to leucocyte suspensions in similar concentration. All spermatozoa/leucocyte suspensions were then incubated at $37^{\circ} \mathrm{C}$ for $12 \mathrm{hr}$ in Carrel flasks mounted upon a rocking device.

Six pseudopregnant females, which 8 to 12 days previously had received an 
intravenous injection of 50 i.u. human chorionic gonadotrophin, were inseminated intravaginally with $1.5 \mathrm{ml}$ ejaculate collected from three fertile males and pooled before insemination. In addition two pseudopregnant females were anaesthetized with Nembutal and, following laparotomy, approximately 2 million spermatozoa from the vas deferens in $0 \cdot 1 \mathrm{ml}$ Ringer were instilled through the cervix into each uterine horn. These females were killed $12 \mathrm{hr}$ after insemination and the uterine content collected as described above.

Eight other oestrous females were anaesthetized and laparotomy performed. Approximately 30 million spermatozoa in $0.25 \mathrm{ml}$ Ringer solution from the vas deferens and cauda epididymis were instilled into one pleural cavity by injection through the central membranous part of the diaphragm. By using this route the amount of blood entering the cavity was minimized. At the same time 50 to 100 million spermatozoa in $1 \mathrm{ml}$ serum-Ringer were inseminated intravaginally. The females were killed $12 \mathrm{hr}$ later by dislocation of the neck and fluid was collected from the thoracic cavity. The uterine contents were recovered as described above.

All samples were prepared for microscopy as follows: after centrifugation at $1500 \mathrm{rev} / \mathrm{min}$ for $15 \mathrm{~min}$, the supernatant fluids were decanted and the precipitate pellets containing spermatozoa and leucocytes were fixed for $2 \mathrm{hr}$ at $5^{\circ} \mathrm{C}$ in $1 \%$ osmium tetroxide in veronal acetate buffer, $\mathrm{pH} 7.4$ (Palade, 1952), with the addition of $0 \cdot 25 \mathrm{M}$-sucrose (Caulfield, 1957). After fixation the pellets were immersed in $30 \%$ methanol and were dehydrated in ascending concentrations of methanol up to $100 \%$ during a period of $1 \mathrm{hr}$, followed by immersion in acetone for $2 \mathrm{hr}$. After embedding in araldite (Glauert \& Glauert, 1958), sections cut to $0.08 \mu$ thickness were stained with lead hydroxide (Millonig, 1961) or with uranyl acetate and potassium permanganate. The sections were examined with an EMU 3F (R.C.A.) electron microscope.

\section{RESULTS}

INGESTION OF SPERMATOZOA IN THE OESTROUS UTERUS

The plasma membrane remains intact in most spermatozoa recovered from the uterus of oestrous females 10 to $12 \mathrm{hr}$ after mating (Bedford, 1964). While many of these spermatozoa appear quite free, others are adherent to or are being engulfed by uterine polymorphonuclear leucocytes. In one sample taken at random, for instance, $30 \%$ of 320 uterine spermatozoa were noted to be at some definite stage of ingestion by leucocytes.

In the initial phase of the ingestion process the cell membrane invaginates over the point of contact with the plasma membrane of the sperm head (Pl. 1, Fig. 1). Subsequently the invagination usually develops as a wide cleft which is wholly or partially filled by the swollen plasma membrane of the anterior region of the sperm head (Pl. 1, Fig. 2). In occasional examples, but less commonly, the plasma membrane remains in close apposition to the underlying acrosome cap; in these instances and in cases in which the head membrane and acrosome cap were absent (Pl. 1, Fig. 3), the sperm head is drawn into the leucocyte within a narrow fissure. After total ingestion of the intact sperm head, the anterior region of the head appears to be surrounded by a discrete vacuole (Pl. 1, 
Fig. 4) which in reality is a space existing between the plasma membrane of the sperm head and the underlying acrosome cap. At higher magnification it can be seen that the plasma membrane of the sperm head retains its integrity and identity (Pl. 2, Fig. 5); although completely separate from the acrosome cap the sperm cell membrane remains closely applied to the region of the post-nuclear cap. In no case was any fusion between sperm and leucocyte membranes observed, nor was there evidence of lytic destruction of the membranes of the intact ingested sperm head within $12 \mathrm{hr}$ of mating. In all samples from oestrous uteri a few damaged spermatozoa which had lost the outer membrane and substance of the acrosome cap were observed within leucocytes (Pl. 2, Fig. 6). Formation of pseudopodia by the engulfing phagocyte was rarely seen during ingestion of the head region, but was sometimes observed during ingestion of the tail (Pl. 2, Fig. 7). It is conceivable that this difference may be related to the surface area of the object being ingested, the size of which might temporarily influence the tension of the surface of the phagocytic cell.

\section{INGESTION OF SPERMATOZOA DURING IN-VITRO INGUBATION}

Samples were prepared following in-vitro incubation of epididymal and washed ejaculate spermatozoa in the hope that it might be possible to observe and compare the mode of phagocytosis of these non-capacitated spermatozoa with that of spermatozoa in the oestrous uterus, particularly with respect to the disposition of the plasma membrane overlying the acrosome cap. Rather unexpectedly such comparisons proved impossible. Sections taken through various regions of the fixed materials were examined thoroughly, but after $12 \mathrm{hr}$ incubation, no sperm head with intact plasma membrane was ever observed partially or completely within a leucocyte. Many intact spermatozoa were adherent to the surface of leucocytes, and in some instances invagination of the surface had commenced at the point of sperm contact; in no case, however, had ingestion proceeded further than the initial phase seen in Pl. 2, Fig. 8. The same sections revealed spermatozoa which lacked the acrosome cap membranes and/or the plasma membrane and which were being or had been completely ingested, thus presenting a similar picture to that seen in Pl. 1, Fig. 3 and Pl. 2, Fig. 6. One or two cases were observed in which the acrosome cap had remained intact in an ingested spermatozoon in which the plasma membrane was absent.

\section{EXPLANATION OF PLATE 1}

FIG. 1. Section showing the initial indentation of the cell membrane of a leucocyte in the oestrous uterus in response to the presence of the membrane of the sperm head. Lead hydroxide. $\times 29,000$.

FIG. 2. Later stage of the ingestion process in the oestrous uterus. Note the wide cleft partially occupied by the swollen plasma membrane overlying the acrosome cap. Lead hydroxide. $\times 16,500$.

Fig. 3. Section showing partially ingested sperm head in which both plasma membrane and acrosome cap are missing. Taken after $12 \mathrm{hr}$ in-vitro incubation with leucocytes from oestrous uterus. Note the narrow fissure into which the damaged sperm head is being drawn. Black bodies are ingested charcoal granules. Uranyl acetate and potassium permanganate. $\times 31,000$.

FIG. 4. Two leucocytes which have completely engulfed the heads of intact spermatozoa in the oestrous uterus. Note the intact swollen membrane of the sperm head, which forms a vesicle around the acrosome region. Lead hydroxide. $\times 12,600$. 


\section{PLATE 1}
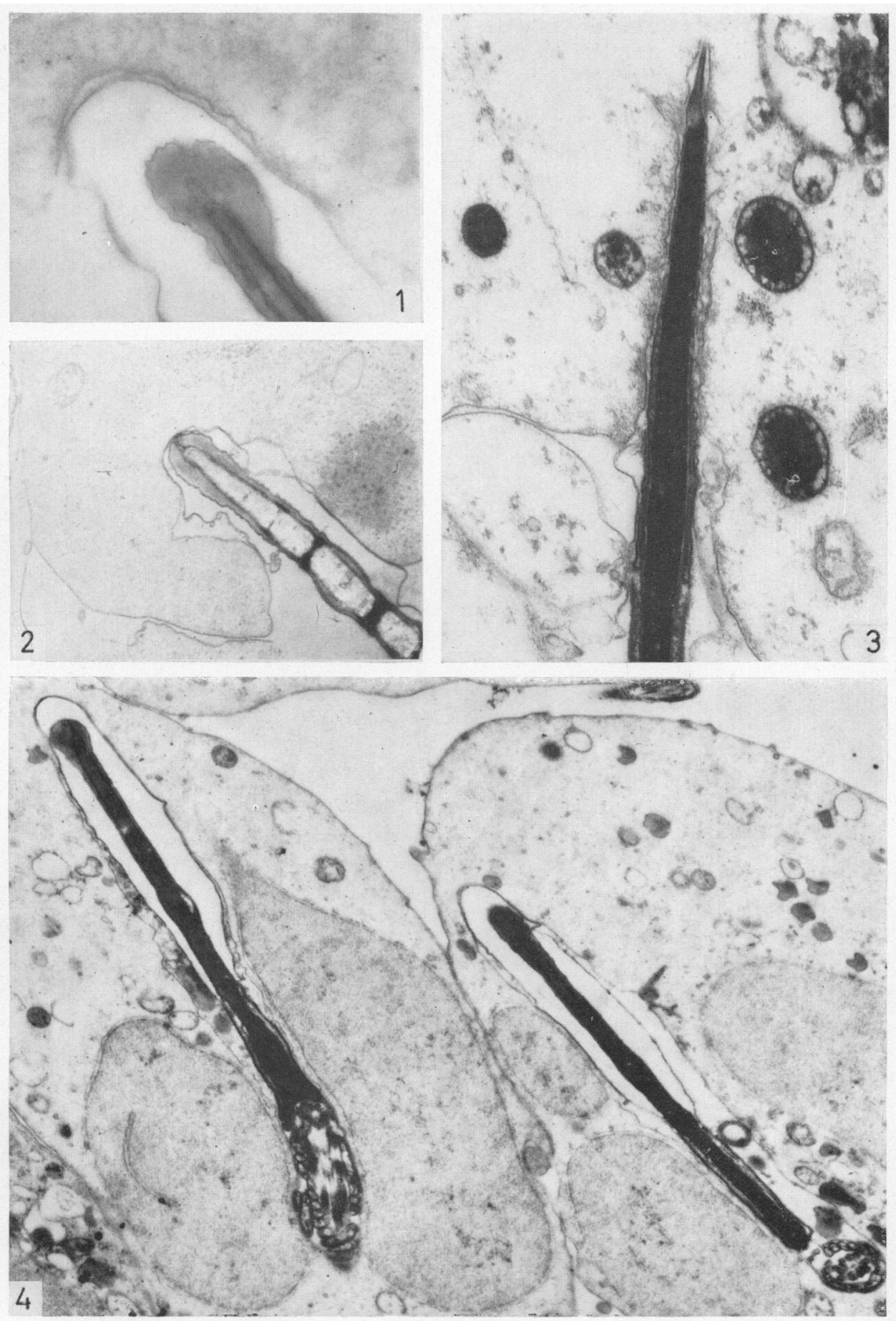

(Facing p. 252) 
PLATE 2
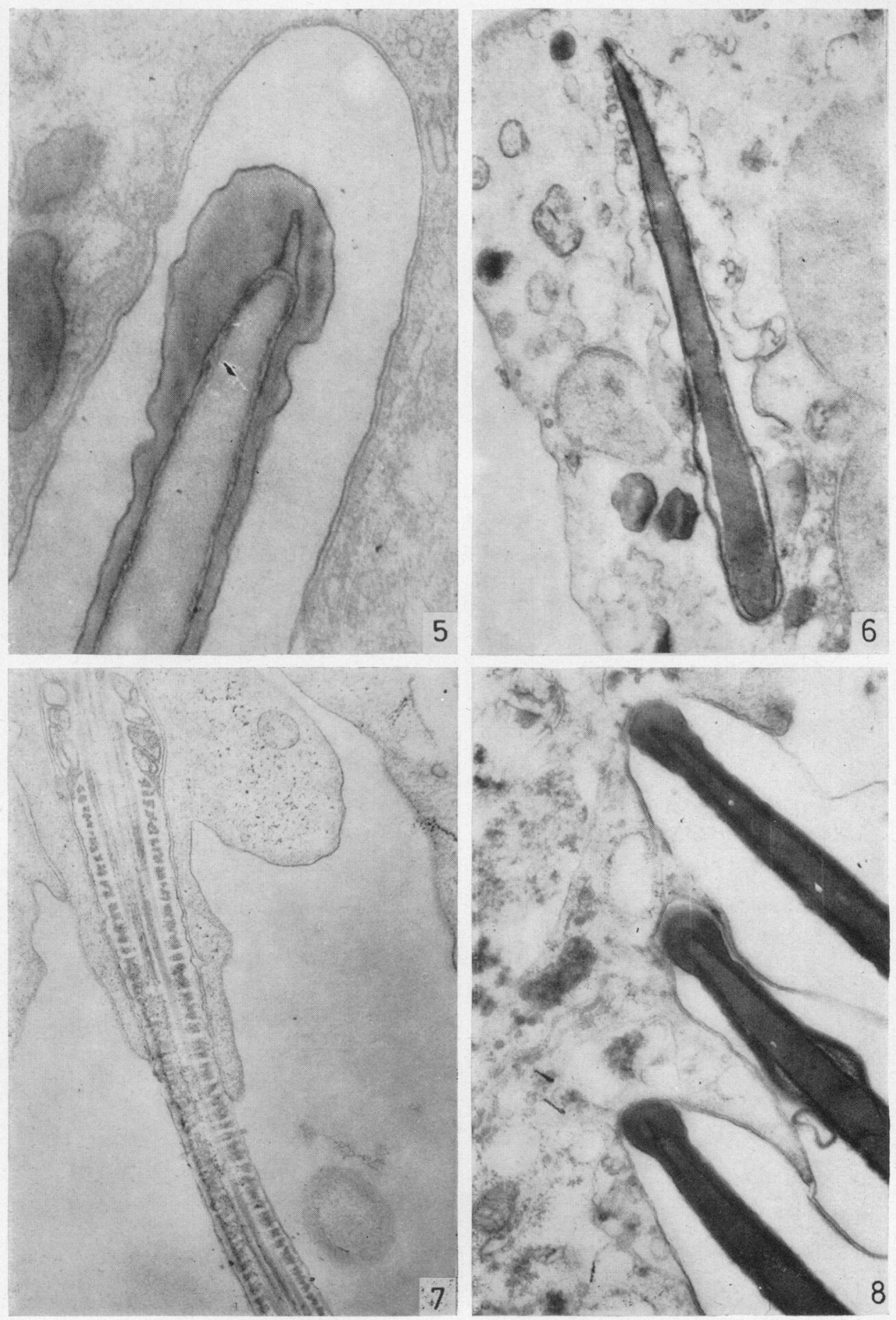
In these latter examples the invaginated leucocyte membrane remained quite distinct and it is certain that the plasma membrane of the sperm head had not been lysed after ingestion. The fact that after such in-vitro incubation several leucocytes were observed to contain as many as three or four damaged sperm heads serves to emphasize that the phagocytic activity of the leucocytes was not impaired during the incubation period.

\section{INGESTION OF SPERMATOZOA IN THE PSEUDOPREGNANT UTERUS}

Most spermatozoa in flushings recovered from pseudopregnant uteri appeared intact and morphologically quite normal. In all samples, from which hundreds of spermatozoa were examined, only one intact sperm was seen in which about two-thirds of the head had been ingested by a leucocyte. Many intact sperm heads were adherent to the surface of leucocytes and in some of these the cell membrane had become completely separated from the underlying acrosome cap. Many damaged sperm heads were observed within or in the process of ingestion by uterine leucocytes.

\section{INGESTION OF SPERMATOZOA IN THE PLEURAL GAVITY}

Most spermatozoa recovered after $12 \mathrm{hr}$ incubation in the pleural cavity had lost the cell membrane covering the anterior head region, and exhibited varying degrees of acrosome degeneration. Many of these had been ingested by leucocytes, but no spermatozoa with intact membranes had been engulfed, though some were adherent to leucocyte surfaces. In marked contrast, those spermatozoa taken originally from the same samples, but inseminated intravaginally and recovered from the uterus of the same animal $12 \mathrm{hr}$ later, were generally quite intact and in some instances had been engulfed by leucocytes, as shown in Pl. 1, Fig. 4. This observation, though not unexpected, serves to emphasize the relative suitability of the uterine lumen as an environment for active spermatozoa.

A synopsis of these results is presented in Table 1.

\section{DISCUSSION}

The phagocytic ingestion of intact sperm heads occurs commonly in the oestrous uterus and appears to result simply from an invagination of the leucocyte surface initiated at the point of sperm adhesion. Morphological evidence that attachment of many spermatozoa to leucocytes is a simple state of adhesion between

EXPLANATION OF PLATE 2

Frg. 5. Higher magnification of completely ingested intact sperm head in the oestrous uterus. Note that the plasma membrane of the sperm head which has become completely separated from the underlying acrosome lines the vesicle surrounding the sperm head, and remains in close apposition to the leucocyte cell membrane. Lead hydroxide. $\times 60,000$.

Fig. 6. Spermatozoon without plasma membrane or acrosome cap, ingested in the oestrous uterus. Lead hydroxide. $\times 16,500$.

FIG. 7. Sperm tail immediately distal to the midpiece. Note the pseudopodia extending from the engulfing leucocyte along the sperm tail. Lead hydroxide. $\times 26,000$.

FIG. 8. Beginnings of invagination of the leucocyte surface in response to spermatozoa after $12 \mathrm{hr}$ incubation in vitro. Note that the swollen plasma membrane of the sperm head follows closely the contours of the invaginated leucocyte cell surface. Uranyl acetate and potassium permanganate. $\times 26,000$. 
surface membranes is supported by experiments (unpublished), in which the left and right uterine horns were flushed with solutions of $0.145 \mathrm{~m}-\mathrm{NaCl}$ and $0 \cdot 3 \mathrm{~m}$-fructose, respectively, some $12 \mathrm{hr}$ after mating. Immediate examination showed that motile spermatozoa in the saline flush were associated with leucocytes, whereas a majority of spermatozoa in the fructose flush were swimming freely with only a few adhering to leucocytes in the medium. This finding is in accord with the observation that the tendency of sperm heads to adhere becomes minimal in media having very low concentrations of free ions. In most though not all intact spermatozoa being ingested, the cell membrane overlying the acrosome appeared swollen. In these cases the invagination of the leucocyte surface was correspondingly wide, in contrast to the narrow cleft surrounding ingested sperm heads in which the surface membranes had been lost prior to ingestion. While such membrane swelling might perhaps be regarded as an artefact of fixation, pictures of various stages of ingestion of intact

TABLE 1

\begin{tabular}{c|l|c|c}
\hline \multirow{2}{*}{ No. animals } & \multicolumn{1}{|c}{ Sperm incubation site } & $\begin{array}{c}\text { Phagocytosis } \\
\text { membranes } \\
\text { intact }\end{array}$ & $\begin{array}{c}\text { Sperm head } \\
\text { membranes absent } \\
\text { or damaged }\end{array}$ \\
\hline 16 & $\begin{array}{l}\text { Oestrous uterus } \\
\text { In vitro with leucocytes } \\
\text { from oestrous uterus }\end{array}$ & + & + \\
8 & $\begin{array}{l}\text { Pseudopregnant uterus } \\
\text { (8 to 12 days) } \\
\text { Pleural cavity of oestrous } \\
\text { female }\end{array}$ & - & + \\
8 & - & + \\
\hline
\end{tabular}

spermatozoa by uterine leucocytes do suggest that this anterior head membrane may often swell in vivo, before fixation.

There is clearly an essential difference of detail between the mode of sperm entry into the vitellus and that of uterine phagocytosis. The membranes surrounding the sperm nucleus in the perivitelline space apparently lose their identity during entry into the vitellus, possibly by fusion with the vitelline surface (Szollosi \& Ris, 1961; Piko \& Tyler, 1964); engulfment of the intact or damaged sperm head by leucocytes is completed without obvious change in identity of sperm or leucocyte surface membranes (PI. 2, Fig. 5).

It was not possible, as originally intended, to compare the disposition of the surface membrane of non-capacitated spermatozoa after ingestion by phagocytes. However, the obvious discrimination exercised by leucocytes against intact spermatozoa in all situations except that of the oestrous uterus raises questions of perhaps greater significance. It seems unlikely that differences in phagocytic activity towards spermatozoa in various experimental situations could have been due only to differences inherent in the leucocytes themselves. Leucocytes used for in-vitro incubation were recovered from oestrous uteri in a small amount of fluid and in all cases showed considerable phagocytic activity towards spermatozoa whose surface structures were damaged. It has been shown that the phagocytic activity of leucocytes towards starch particles is reduced in the 
pseudopregnant uterus (Killingbeck \& Lamming, 1963), yet in the present experiments many damaged spermatozoa were observed to have been ingested by phagocytes in the uteri of pseudopregnant animals. It is apparent that residence in the pleural cavity for any length of time results in marked damage to the acrosome cap in most spermatozoa, many of which are then ingested. In no case, however, were spermatozoa with intact cell membranes phagocytized in the pleural cavity.

The ingestion by non-sensitized polymorphonuclear leucocytes of whole intact cells from an homologous species would seem to be a somewhat surprising occurrence. It does not seem justified to consider intact spermatozoa phagocytized in the oestrous uterus merely as aged or effete cells, as it is equally likely that such effete cells would also occur in any of the situations considered here. It is also worthy of note that spermatozoa taken from oestrous uteri after mating may sometimes be observed, by means of a phase microscope, being phagocytized while still retaining active motility.

The fineness of discrimination by phagocytes is not often appreciated; present views are in accord with the idea that the high degree of specificity in the responses of phagocytes can be accounted for only by assuming that phagocytic discrimination is dependent upon differences in structure between familiar surface macromolecules, and unfamiliar macromolecules of foreign or injured cells (Boyden, 1963). In this instance it seems reasonable to suggest that the vital surface membrane of the sperm head is altered in some way while resident in the oestrous uterus; such change might be brought about by specific enzymatic removal of some surface component, or by the adsorption of a particular opsonin which would facilitate specific interaction between host components adsorbed on the sperm surface and components of the leucocyte surface.

In the light of these findings, it seems possible that electron microscopic studies of the reactions of leucocytes might be useful for detecting differences in sperm, and possibly in surface membranes of other cell types.

The failure to find any striking morphological change in the uterine sperm head (Bedford, 1964), and the fact that the state of capacitation may be reversibly inhibited by some component present in seminal plasma (Chang, 1957; Bedford \& Chang, 1962), indicate that some phase of the capacitation process in rabbit spermatozoa may well be concerned with change in the sperm surface. Although rabbit spermatozoa may easily be capacitated in the oestrous uterus, capacitation does not occur under these in-vitro conditions, or in the pseudopregnant uterus (Chang, 1958). In addition it has not proved possible, contrary to inferences drawn from the results of Noyes, WaIton \& Adams (1958), to obtain functional capacitation of rabbit spermatozoa in the pleural cavity (unpublished). These facts, together with results presented in this report, point to the possibility that the change in spermatozoa in the oestrous uterus which renders them acceptable to leucocytes may also constitute one phase of the capacitation phenomenon.

\section{ACKNOWLEDGMENTS}

Supported in part by U.S. Public Health Service grant R.G. 6489 from National Institutes of Health, Bethesda, Maryland. 


\section{REFERENCES}

Adams, C. E. \& Chang, M. C. (1962) Capacitation of rabbit spermatozoa in the Fallopian tube and uterus. F. exp. Zool. 151, 159.

Austin, G. R. (1951) Observations on the penetration of sperm into the mammalian egg. Aust. $\mathcal{J}$. sci. Res. B, 4, 481.

Austin, C. R. (1957) Fate of spermatozoa in the uterus of the mouse and rat. F. Endocrin. 14, 335.

Austin, C. R. (1963) Acrosome loss from the rabbit spermatozoon in relation to entry into the egg. $\mathcal{F}$. Reprod. Fertil. 6, 313.

BEDFORD, J. M. (1963) Morphological reaction of spermatozoa in the female reproductive tract of the rabbit. F. Reprod. Fertil. 6, 245.

BEDFoRd, J. M. (1964) Fine structure of the sperm head in ejaculate and uterine spermatozoa of the rabbit. F. Reprod. Fertil. 7, 221.

Bedford, J. M. \& Chang, M. C. (1962) Removal of decapacitation factor from seminal plasma by high speed centrifugation. Amer. 7. Physiol. 202, 179.

Boyden, S. (1963) Cellular recognition of foreign matter. Int. Rev. exp. Path. 2, 311.

Gaulfield, J. B. (1957) Effects of varying the vehicle for $\mathrm{OsO}_{4}$ in tissue fixation. 7. biophys. biochem. Cytol. 3, 827.

Chang, M. G. (1951) Fertilising capacity of spermatozoa deposited into the Fallopian tubes. Nature, Lond. 168, 697.

Ghang, M. G. (1957) A detrimental effect of seminal plasma on the fertilising capacity of sperm. Nature, Lond. 179, 258.

Chang, M. C. (1958) Capacitation of rabbit spermatozoa in the uterus with special reference to the reproductive phases of the female. Endocrinology, 63, 619.

Glauert, A. M. \& Glauert, R. H. (1958) Araldite as an embedding medium for electron microscopy. 7. biophys. biochem. Cytol. 4, 191.

HAMNER, C. E. \& Williams, W. L. (1963) Effects of the female reproductive tract on sperm metabolism in the rabbit and fowl. F. Reprod. Fertil. 5, 143.

KillingBeck, J. \& Lamming, G. E. (1963) Influence of uterine secretions on phagocytosis. Nature, Lond. 198, 117.

Mrllonig, G. (1961) A modified procedure for lead staining of thin sections. F. biophys. biochem. Cytol. 3,827 .

Mounib, M. S. \& Chang, M. C. (1964) Effect of in utero incubation on the metabolism of rabbit spermatozoa. Nature, Lond. 201, 943.

Noyes, R. W., Walton, A. \& AdAms, C. E. (1958) Capacitation of rabbit spermatozoa. F. Endocrin. 17,374 .

Palade, G. E. (1952) A study of fixation for electron microscopy 7. exp. Med. 95, 285.

Piko, L. \& Tyler, A. (1964) Fine structural studies of sperm penetration in the rat. Proc. Vth int. Congr. Anim. Reprod., Trento, 2, p. 372.

Szollosi, D. \& Ris, H. (1961) Observations on sperm penetration in the rat. F. biophys. biochem. Cytol. 10,275 . 